\title{
COUPLED ADJOINT FLUID-STRUCTURE INTERACTION TECHNIQUE FOR FLEXIBLE WING SHAPE OPTIMIZATION
}

\author{
K. TSIAKAS ${ }^{1}$, G. ROGÉ ${ }^{2}$, X. TROMPOUKIS ${ }^{1}$, V. ASOUTI ${ }^{1}$, G. FOUGERON TRO $^{3}$, \\ S. JULISSON ${ }^{2}$, L. MARTIN ${ }^{2}$, S. KLEINVELD ${ }^{2}$ AND K. GIANNAKOGLOU ${ }^{1}$ \\ ${ }^{1}$ Parallel CFD \& Optimization Unit (PCOpt), School Of Mechanical Engineering, \\ National Technical University of Athens (NTUA), 9, Iroon Polytechniou, 15780, Zografou, Greece \\ \{tsiakost, xeftro\}@gmail.com, \{vasouti, kgianna\}@mail.ntua.gr \\ ${ }^{2}$ Dassault Aviation \\ 78, quai Marcel Dassault, 92552, Saint-Cloud, France \\ \{gilbert.roge, sarah.julisson, ludovic.martin, steven.kleinveld\}@ dassault-aviation.com \\ ${ }^{3}$ ESI Group, 3 Rue Saarinen, 94150 Rungis, France \\ gabriel.fougeron@esi-group.com
}

Key words: Aeroelasticity, Adjoint Method, Multi-disciplinary Optimization

\begin{abstract}
This paper presents the Multi-Disciplinary Optimization (MDO) of a business jet including static aeroelastic effects. Two different CFD tools (AETHER by Dassault Aviation and PUMA by NTUA) are coupled with a CSM model (VPS software by ESI). Single discipline as well as coupled multi-disciplinary sensitivity derivatives (SDs) are computed by means of adjoint methods. Both a purely discrete and a hybrid continuous(fluid)/discrete(structure) adjoint formulations are presented. The computed SDs are verified against finite differences.
\end{abstract}

\section{INTRODUCTION}

Modern aircraft design is an increasingly complex process due to stricter regulations, environmental constraints and a continuous need for lower operational costs. Design includes aerodynamics, structural mechanics, control, aeroacoustics and heat transfer, to name just the most important disciplines. There is a large literature on simulation tools for these disciplines, the use of which has led to efficient and increasingly versatile aircraft. A major challenge for future design workflows, especially those relying on numerical optimization techniques, is the exploitation of trade-offs between the involved disciplines which is expected to reduce the development effort, resulting in superior new designs.

Among the variety of optimization techniques used by the aeronautics industry, a significant amount of interest has surfaced in gradient-based techniques relying upon the adjoint method $[1,2,3]$, since the latter computes gradients for aircraft performance metrics at a cost independent of the number of design parameters. However, adjoint formulations that account for the interaction between disciplines, are not yet mature enough and this is where recent research focuses on $[4,5]$. The development of adjoint methods in an MDO context gives the opportunity to obtain feasible designs at a fraction of the development cost compared to traditional methods, iteratively solving single-discipline optimization problems. The latter are more costly yielding inferior results, since the multi-disciplinary feasibility must 
a posteriori be enforced for each candidate design.

In this work, adjoint methods for tackling the problem of aircraft wing design while also considering the wing's structure flexibility are developed. These make use of coupled high-fidelity computational tools (for the Reynolds-Averaged Navier-Stokes, RANS, equations for the fluid and a Finite Element, FE, solver for the wing structure) to support flexible wing optimization. The developed methods are used for the shape optimization of a Generic Business Jet (GBJ) flexible wing provided by Dassault Aviation.

The rest of this paper is as follows. Section 2 describes the two different CFD solvers, namely AETHER and PUMA, used in this paper. Comparisons of the GBJ aerodynamic performance predictions by them are made. Section 3 describes the adjoint formulations for both CFD tools. AETHER employs discrete adjoint based on Automatic Differentiation (AD), while PUMA continuous adjoint. Section 4 describes the approach used for parameterizing the shape of the GBJ. A surrogate parameterization model provides an opaque layer of abstraction between the actual CAD model of the geometry and the optimization process. Section 5 describes the Computational Structural Mechanics (CSM) model used, by the VPS software, as well as the Fluid-Structure Interaction (FSI) technique for both the primal and adjoint workflows. In Section 6, the sensitivity derivatives predicted by the adjoint methods, with and without accounting for wing structure flexibility, 0 are compared with finite differences (FDs). Section 7 presents the outcome of the optimization runs.

\section{CFD TOOLS}

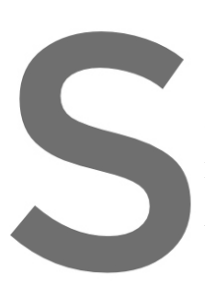

Two CFD tools are sault Aviation. AETHHR approach for solving the marching procedure basec AETHER runs on CPU cliction The second CFD tool is software PUMA [8]
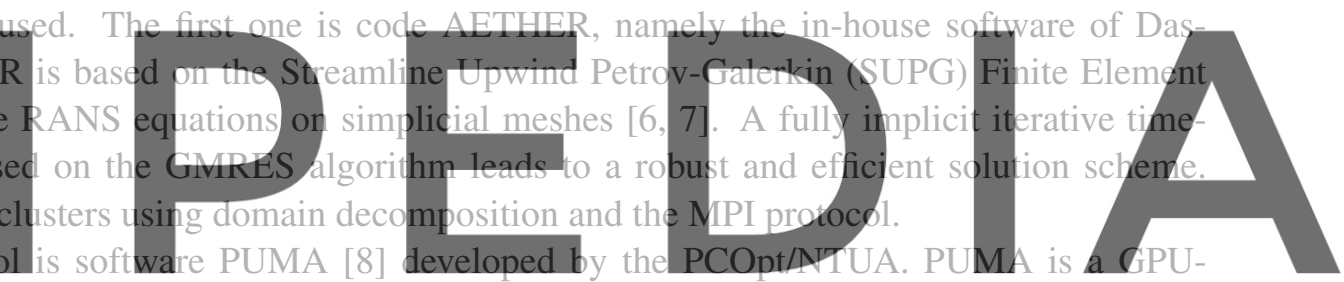

enabled (U)RANS solver, employing the vertex-centered approach of the finite volume method on un-

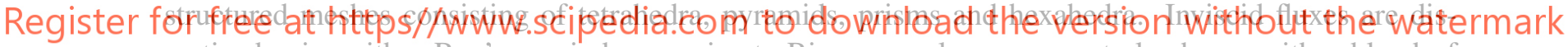
cretized using either Roe's upwind approximate Riemann solver or a central scheme with a blend of second- and fourth-order dissipation terms for second-order spatial accuracy. It employs a multi-stage

Runge-Kutta scheme with residual smoothing, for which the linear system is solved by a preconditioned Krylov-based solver. PUMA runs on clusters of GPUs on different computational nodes, by decomposing the flow domain into overlapping subdomains. GPUs on the same node exchange data through the shared on-node memory using the capabilities of the CUDA API, while the MPI protocol is used otherwise.

A comparison of the $c_{L}$ vs. $c_{D}$ curves for a cruise speed of $M=0.82$ and a flight altitude of $41000 \mathrm{ft}$, as computed by the two CFD tools, is presented in Fig. 2. The two curves match each other and the comparison is very satisfactory given that the two RANS solvers rely upon different methods (FE vs. FV) and meshes. AETHER used a tetrahedral mesh created by in-house Dassault Aviation tools with approximately $16 \mathrm{Mi}$ nodes while PUMA used a hybrid mesh of 9 Mi nodes, Fig. 1. In either code, turbulence is modeled by means of the Spalart-Allmaras model [9]. 


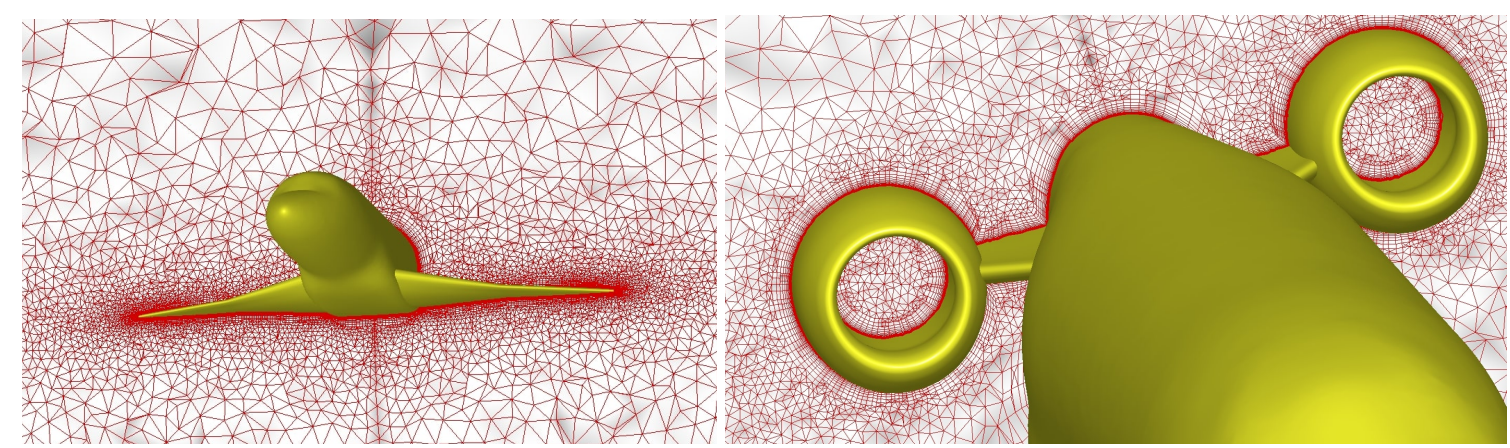

Figure 1: Cut across the volume mesh around the GBJ used by PUMA (left). Hexahedra and prisms are used for creating high aspect ratio cells to capture the boundary layer physics. Tetrahedra fill the rest of the domain, while pyramids are used to transition between the different element types. Close-up view of the mesh in the nacelle-pylon-fuselage area (right).
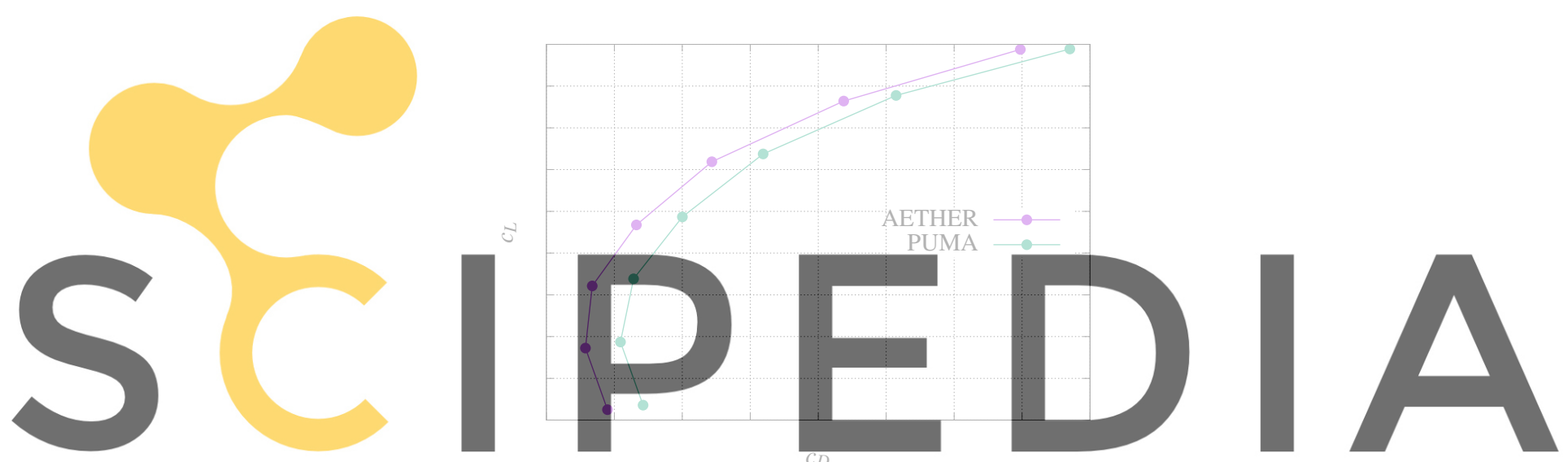

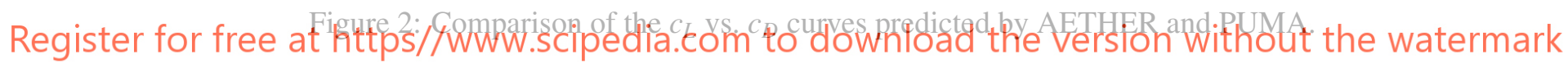

\section{ADJOINT FORMULATIONS}

The two CFD tools (PUMA and AETHER) employ different adjoint approaches. In the discrete adjoint approach, the expression of the objective or constraint function $J$ is augmented by the discrete residual of the flow (index $F$ ) equations $\boldsymbol{R}_{n}^{F}$, multiplied by the vector of adjoint variables $\Psi_{n}^{F}$, with $n=1, \ldots, M^{F}$, with $M^{F}$ the number of the flow equations. Boldface symbols denote vectors with size equal to that of the computational mesh. By differentiating the expression of $J_{\text {aug }}$ w.r.t. the design parameters $b_{i}, i=1, \ldots, N^{D}$ the following expression arises

$$
\frac{\delta J_{\mathrm{aug}}}{\delta b_{i}}=\frac{\delta J}{\delta b_{i}}+\left[\boldsymbol{\Psi}_{n}^{F}\right]^{T} \frac{\delta \boldsymbol{R}_{n}^{F}}{\delta b_{i}}
$$

Throughout this paper, repeated indices imply summation, unless otherwise stated. Since both $J$ and $\boldsymbol{R}_{n}^{F}$ are functions of the flow variables $\boldsymbol{W}_{m}^{F}, m=1, \ldots, M^{F}$ and nodal coordinates $\boldsymbol{x}_{k}^{F}, k=1, \ldots, 3$, their 
variations w.r.t. $b_{i}$ are

$$
\frac{\delta J}{\delta b_{i}}=\frac{\partial J}{\partial \boldsymbol{W}_{m}^{F}} \frac{\delta \boldsymbol{W}_{m}^{F}}{\delta b_{i}}+\frac{\partial J}{\partial \boldsymbol{x}_{k}^{F}} \frac{\delta \boldsymbol{x}_{k}^{F}}{\delta b_{i}}, \quad \frac{\delta \boldsymbol{R}_{n}^{F}}{\delta b_{i}}=\frac{\partial \boldsymbol{R}_{n}^{F}}{\partial \boldsymbol{W}_{m}^{F}} \frac{\delta \boldsymbol{W}_{m}^{F}}{\delta b_{i}}+\frac{\partial \boldsymbol{R}_{n}^{F}}{\partial \boldsymbol{x}_{k}^{F}} \frac{\delta \boldsymbol{x}_{k}^{F}}{\delta b_{i}}
$$

By combining Eq. 1 with Eq. 2 and re-arranging terms, one obtains

$$
\frac{\delta J_{\text {aug }}}{\delta b_{i}}=\left(\frac{\partial J}{\partial \boldsymbol{W}_{m}^{F}}+\left[\boldsymbol{\Psi}_{n}^{F}\right]^{T} \frac{\partial \boldsymbol{R}_{n}^{F}}{\partial \boldsymbol{W}_{m}^{F}}\right) \frac{\delta \boldsymbol{W}_{m}^{F}}{\delta b_{i}}+\left(\frac{\partial J}{\partial \boldsymbol{x}_{k}^{F}}+\left[\boldsymbol{\Psi}_{n}^{F}\right]^{T} \frac{\partial \boldsymbol{R}_{n}^{F}}{\partial \boldsymbol{x}_{k}^{F}}\right) \frac{\delta \boldsymbol{x}_{k}^{F}}{\delta b_{i}}
$$

Variations in the flow variables are eliminated by satisfying the discrete adjoint equations (i.e. $\left[\frac{\partial \boldsymbol{R}_{n}^{F}}{\partial \boldsymbol{W}_{m}^{F}}\right]^{T} \boldsymbol{\Psi}_{n}^{F}=$ $\left.-\left[\frac{\partial J}{\partial W_{m}^{F}}\right]^{T}\right)$, while the second term on the r.h.s. of Eq. 3 provides the sensitivity derivatives' expression.

The discrete adjoint approach, used by AETHER [10] is based on the AD tool TAPENADE [11]. The Spalart-Allmaras turbulence model is also included in the AD process, hence, $\boldsymbol{R}^{F}$ also accommodates the residual of the discretized Spalart-Allmaras model equation.

In order to compute SDs using continuous adjoint, $J$ is augmented by the integrals of the flow equations $R^{F}$ multiplied by the adjoint variables $\Psi^{F}$. No boldface symbols are used since the adjoint development is agnostic to any domain discretization. Development starts from

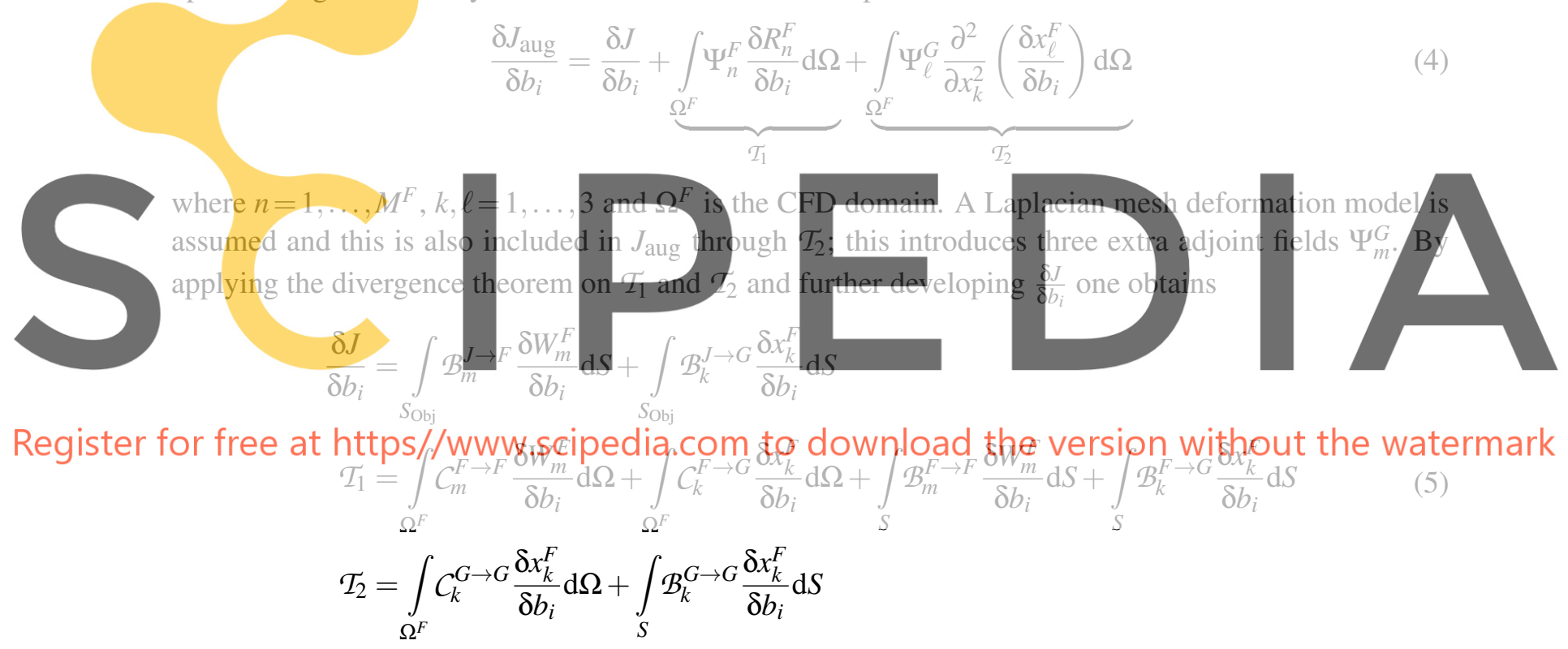

where $S$ stands for the boundary of $\Omega, S_{\text {obj }}$ the surface on which $J$ is defined, $m=1, \ldots, M^{F}$ and $k=1, \ldots, 3$. Combining Eqs. 5 and satisfying (a) the adjoint flow equations $C_{m}^{F \rightarrow F}=0$ with appropriate boundary conditions, i.e. $\mathcal{B}_{m}^{J \rightarrow F}+\mathcal{B}_{m}^{F \rightarrow F}=0$ and (b) the adjoint grid displacement equations $C_{k}^{F \rightarrow G}+\mathcal{C}_{k}^{G \rightarrow G}=0$, the derivatives of $J$ w.r.t. $b_{i}$ are computed as

$$
\frac{\delta J}{\delta b_{i}}=\int_{S}\left(\mathcal{B}_{k}^{J \rightarrow G}+\mathcal{B}_{k}^{F \rightarrow G}+\mathcal{B}_{k}^{G \rightarrow G}\right) \frac{\delta x_{k}^{F}}{\delta b_{i}} \mathrm{~d} S
$$

In the adjoint PUMA code, the Spalart-Allmaras turbulence model is fully differentiated $[12,13]$. Possible numerical stability issues of the adjoint solver due to the presence of unstable modes in the primal solution, are circumvented using the Recursive Projection Method (RPM) [14]. 


\section{GBJ SHAPE PARAMETERIZATION}

The shape of the airframe is parameterized using the geometry generation tool GANIMEDE (Geometry ANd Inherent MEsh DEformation, [15, 16]), by Dassault Aviation. GANIMEDE handles both local and global design variables. By local design variables modifications at the control point level are meant, such as coordinates', tangent and curvature values. Global design variables redefine several control points enabling to modify characteristics such as thickness, twist and camber of wing sections. As the geometry changes during the optimization, GANIMEDE generates new surface meshes by involving the CAD modeler and creating connectivity by projecting the initial surface mesh onto the new geometry. Quite often, the need to take geometric constraints into account emerges occasionally. For manufacturing reasons, one might want to impose specific relations between some geometric entities. From the operational point of view, for many aircraft, including very large commercial transport concepts, a span constraint has to be imposed in the design process. Another case of interest for imposing constraints exists when retrofitting an aircraft. All geometric constraints are directly handled by the GANIMEDE modeler. This results to a cost reduction compared to the alternative of delegating this task to the optimizer by imposing additional constraints, since the gradient of these geometric quantities is no longer needed.

Since GANIMEDE is not available for use outside Dassault Aviation, NTUA has developed a surrogate shape parameterization model, working directly on the aircraft's surface mesh, based on meshes corresponding to shape perturbations for each design parameter, provided using GANIMEDE. By doing so, the dependence on GANIMEDE is restricted only to the pre-processing phase, while shape modifications during the optimization

Let $z^{D}$ be the coordin $1, \ldots, N^{D}$ the design par

mesh nodes coordinates

datum values, excluding

positive perturbation of the $i$ th design parameter. 'T
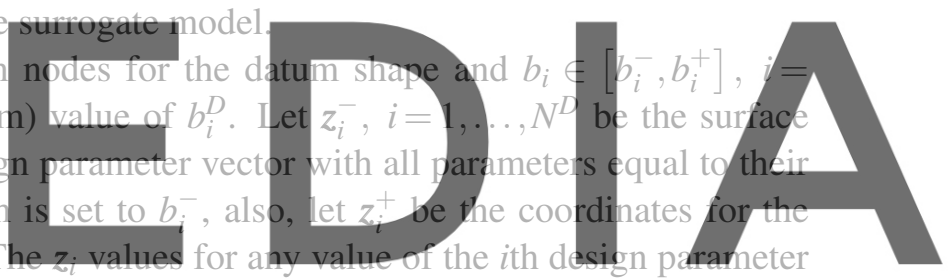

are given by

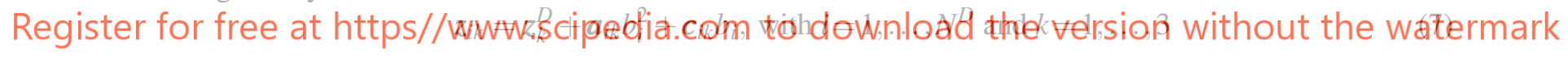

(no summation for repeated indices). The coefficients $a$ and $c$ are computed by solving the following system of equations for each surface mesh node, design parameter and Cartesian coordinate

$$
\begin{aligned}
& \boldsymbol{a}_{i k}\left(b_{i}^{-}\right)^{2}+\boldsymbol{c}_{i k} b_{i}^{-}=z_{i k}^{-}-z_{k}^{D} \\
& \boldsymbol{a}_{i k}\left(b_{i}^{+}\right)^{2}+\boldsymbol{c}_{i k} b_{i}^{+}=z_{i k}^{+}-z_{k}^{D}
\end{aligned}
$$

The surface mesh nodal coordinates $\boldsymbol{x}$ for a simultaneous modification of several design parameters results from superimposing the various $z$ values for all $i=1, \ldots, N^{D}$, i.e.

$$
\boldsymbol{x}_{k}=z_{k}^{D}+\sum_{i=1}^{N^{D}}\left(z_{i k}-z_{k}^{D}\right), \text { for } k=1, \ldots, 3
$$

In what follows, 4 design parameters $\left(b_{1}\right.$ to $\left.b_{4}\right)$ are used to control the wing twist spanwise distribution, another $4\left(b_{5}\right.$ to $\left.b_{8}\right)$ to control the wing trailing edge camber distribution along its span and the last one $\left(b_{9}\right)$ to control the horizontal tail plane (HTP) rotation angle. Since this model is analytically differentiated, it can readily be incorporated into the adjoint method. 


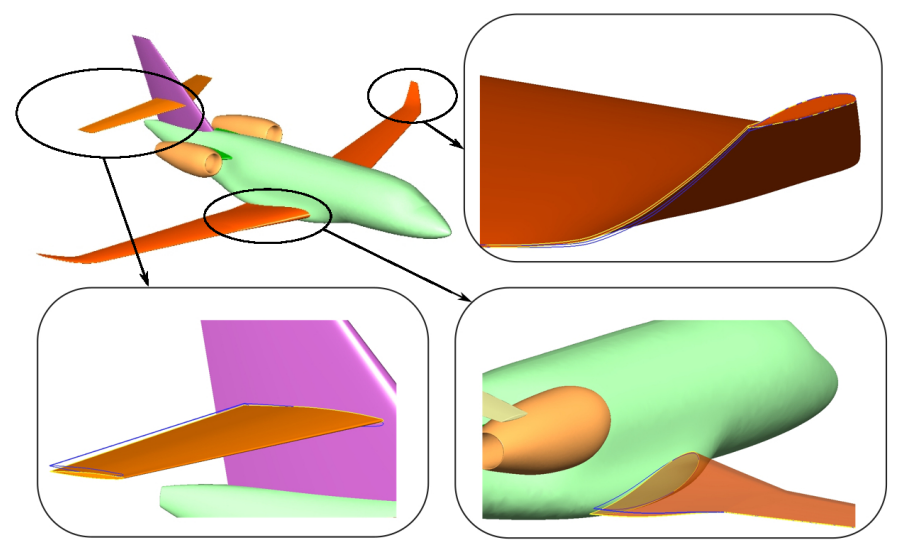

Figure 3: Effect of the design parameters on the airframe shape. The datum contour of the wing is shown with the blue line. The yellow line corresponds to the altered shape outline. Alteration of the $1^{\text {st }}$ (bottom-right), $8^{\text {th }}$ (top-right) and $9^{\text {th }}$ (bottom-left) design parameters.

\section{FLUID-STRUCTURE INTERACTION (FSI)}

The purpose is to introduce wing flexibility effects into the analysis and optimization of an aircraft. The CFD tools and their adjoint counterparts represent only one discipline involved in the MDO problem. The other discipline concerns the structural response of the wing under aerodynamic and gravitational loading. This is handled by a FE linear elasticity sc
software of ESI, which is the CSM Component of th
Given the positions $r_{k}^{S}$ of the $N^{S}$ FE model node
of the same nodes in the unloaded state (jig shap)
$U_{k}^{S}=x_{k}^{S}-y_{k}^{S}, k=1, \ldots, 3$. The discrete system of expressed as

$$
\mathbb{K}_{k \ell} \boldsymbol{U}_{\ell}^{S}=f_{k}^{S}+f_{k}^{G} \text {, with } k, \ell=1, \ldots, 3
$$

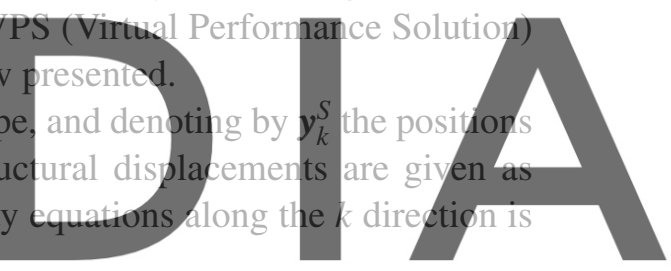

(10)

Register for free at https//www.scipedia.com to download the version without the watermark where $\mathbb{K}$ stands for the stiffness matrix, $f_{k}$ for the aerodynamic load along the cartesian direction applied on the structure nodes and $f_{k}^{G}$ for the gravitational load due to the structure weight (including also the lumped fuel load). Starting from the wing's flight shape, a FE model, consisting of shell elements (for the wing's skin and webs of spars and ribs) and beam elements (for the caps of spars and ribs), is set up. Then, the jig shape of the wing is computed iteratively by a quasi-Newton method, assuming $\mathbb{K}$ to be a function of $y^{S}$.

The CSM mesh differs from the surface CFD mesh. The tool for transferring information along the non-matching interfaces has been developed by ESI, based on Radial Basis Functions (RBFs) [17]. The structural displacements of the CFD surface mesh nodes are given as $\boldsymbol{U}_{k}^{F}=\mathbb{H} \boldsymbol{U}_{k}^{S}, k=1, \ldots, 3$, where matrix $\mathbb{H}$ contains the weights with which each of the $N^{S}$ nodes of the CSM model contributes to the displacement of the $N^{F}$ CFD surface mesh nodes. Aerodynamic load transfer along the FSI is done using the transpose of $\mathbb{H}$, and this ensures conservation of force, moment and virtual work along the FSI [18].

The aeroelastic analysis of the aircraft is performed using a fixed-point iteration scheme with dynamic relaxation [19], sketched in Fig. 4. In order to compute sensitivity derivatives of the aerodynamic performance metrics, while accounting for wing flexibility, the adjoint technique of Section 3 has been extended. Eq. 1 now includes an additional term containing variations in the discrete CSM residual $\left(\frac{\delta \boldsymbol{R}^{S}}{\delta b_{i}}\right)$ 
multiplied by the CSM adjoint variables $\Psi^{S}$, i.e.

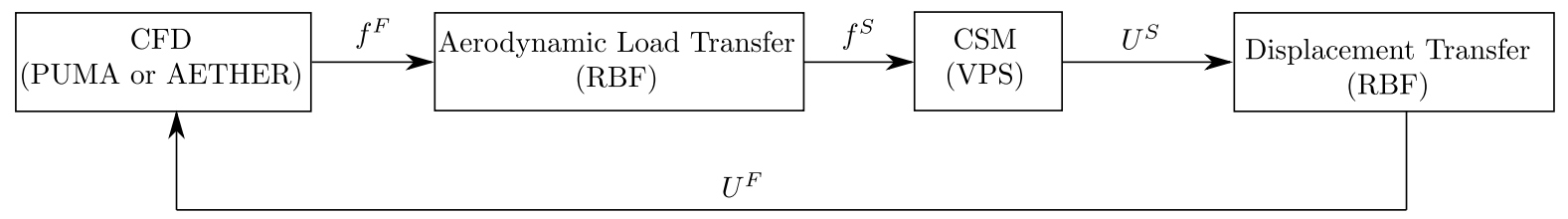

Figure 4: The aeroelastic fixed-point iteration involving the CFD and CSM tools.

$$
\frac{\delta J_{\mathrm{aug}}}{\delta b_{i}}=\frac{\delta J}{\delta b_{i}}+\left[\boldsymbol{\Psi}_{n}^{F}\right]^{T} \frac{\delta \boldsymbol{R}_{n}^{F}}{\delta b_{i}}+\left[\boldsymbol{\Psi}_{k}^{S}\right]^{T} \frac{\delta \boldsymbol{R}_{k}^{S}}{\delta b_{i}}, \text { with } n=1, \ldots, M^{F} \text { and } k=1, \ldots, 3 .
$$

In this work, the wing structure is assumed to remain unaffected by changes in the design parameters. Moreover, the wing's datum flight shape (with nodal coordinates $x^{F_{0}}$ ) is parameterized and will be referred to onward as the "reference" shape. Let $y_{k}^{F}$ denote the coordinates of the wing skin nodes in the unloaded (jig shape) state. Then, the actual flight shape of the wing (when static aeroelastic equilibrium is obtained) is given as $\boldsymbol{x}_{k}^{F}=\boldsymbol{y}_{k}^{F}+\boldsymbol{U}_{k}^{F}, k=1, \ldots, 3$. Due to the assumption made, $\boldsymbol{d}_{k}^{F}=\boldsymbol{x}_{k}^{F_{0}}-\boldsymbol{y}_{k}^{F}, k=1, \ldots, 3$ remain constant and the variation of the flight shape coordinates reads
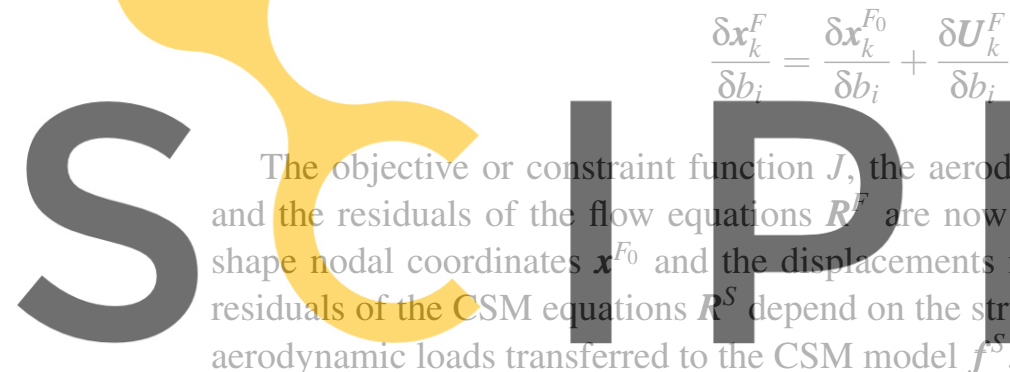
with $k=1, \ldots, 3$

aerodynamic loads transferred to the CSM model $f^{s}$. Eq. 11 then, becomes
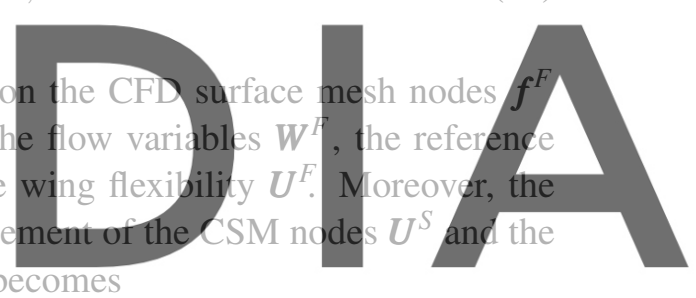

or free at https//www.scipedia.com to download the version without the watermark

$$
\begin{aligned}
\frac{\delta J_{\mathrm{aug}}}{\delta b_{i}} & =\left(\frac{\partial J}{\partial W_{m}^{F}}+\left[\Psi_{n}^{F}\right]^{T} \frac{\partial \boldsymbol{R}_{n}^{F}}{\partial W_{m}^{F}}\right) \frac{\delta W_{m}^{F}}{\delta b_{i}}+\left[\Psi_{k}^{S}\right]^{T} \frac{\partial \boldsymbol{R}_{k}^{S}}{\partial \boldsymbol{U}_{\ell}^{S} \frac{\delta U_{\ell}^{S}}{\delta b_{i}}} \\
& +\underbrace{\left(\frac{\partial J}{\partial \boldsymbol{U}_{\ell}^{F}}+\left[\boldsymbol{\Psi}_{n}^{F}\right]^{T} \frac{\partial \boldsymbol{R}_{n}^{F}}{\partial \boldsymbol{U}_{\ell}^{F}}\right) \frac{\delta \boldsymbol{U}_{\ell}^{F}}{\delta b_{i}}}_{\mathcal{T}_{3}}+\underbrace{\left[\boldsymbol{\Psi}_{k}^{S}\right]^{T} \frac{\partial \boldsymbol{R}_{k}^{S}}{\partial \boldsymbol{f}_{\ell}^{S}} \frac{\delta \boldsymbol{f}_{\ell}^{S}}{\delta b_{i}}}_{\mathcal{T}_{4}}+\left(\frac{\partial \boldsymbol{J}}{\partial \boldsymbol{x}_{\ell}^{F_{0}}}+\left[\boldsymbol{\Psi}^{F}\right]^{T} \frac{\partial \boldsymbol{R}_{n}^{F}}{\partial \boldsymbol{x}_{\ell}^{F_{0}}}\right) \frac{\delta \boldsymbol{x}_{\ell}^{F_{0}}}{\delta b_{i}}
\end{aligned}
$$

with $m, n=1, \ldots, M^{F}$ and $k, \ell=1, \ldots, 3$. The multiplier of the variation of $\boldsymbol{U}^{F}$ is the so-called adjoint structural displacements vector $\left(\boldsymbol{U}^{F, \mathrm{adj}}\right)$ on the CFD surface mesh nodes, while that of the variation of $f^{S}$ represents the adjoint aerodynamic loads $\left(f^{S, \operatorname{adj}}\right)$ on the CSM model nodes. Taking this into account, as well as the equations connecting $\boldsymbol{U}^{F}$ to $\boldsymbol{U}^{S}$ and $\boldsymbol{f}^{S}$ to $\boldsymbol{f}^{F}$, terms $\mathcal{T}_{3}$ and $\mathcal{T}_{4}$ in Eq. 13 become

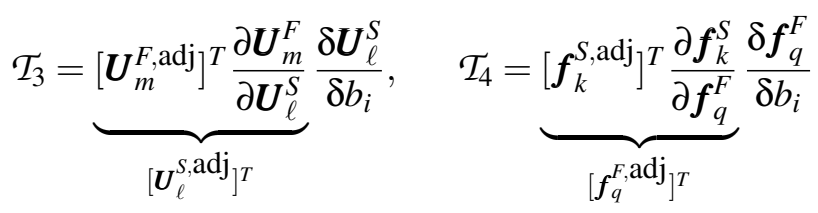


The final expression of the discrete adjoint equations for the coupled CFD+CSM system of equations is

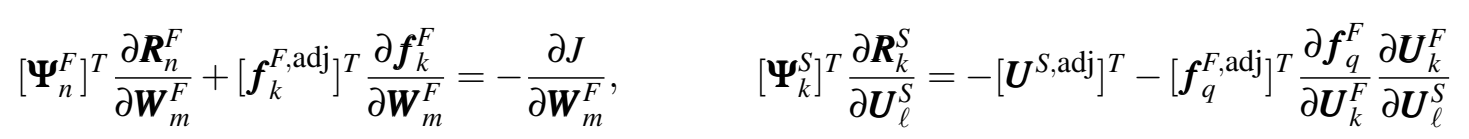

with $m, n=1, \ldots, M^{F}$ and $k, \ell, q=1, \ldots, 3$. Eqs. 15 are solved using the same iterative scheme used for the primal aeroelastic solution. Once $\Psi^{F}$ and $\Psi^{S}$ have been computed, the sensitivity derivatives of $J$ w.r.t. $b_{i}$ are obtained as

$$
\frac{\delta J}{\delta b_{i}}=\left(\frac{\partial J}{\partial x_{k}^{F_{0}}}+\left[\boldsymbol{\Psi}_{m}^{F}\right]^{T} \frac{\partial \boldsymbol{R}_{m}^{F}}{\partial \boldsymbol{x}_{k}^{F_{0}}}+\left[\boldsymbol{f}_{\ell}^{F, \mathrm{adj}^{T}}\right]^{T} \frac{\partial \boldsymbol{f}_{\ell}^{F}}{\partial \boldsymbol{x}_{k}^{F_{0}}}\right) \frac{\delta \boldsymbol{x}_{k}^{F_{0}}}{\delta b_{i}}
$$

with $m=1, \ldots, M^{F}$ and $k, \ell=1, \ldots, 3$, where the variations in $\boldsymbol{x}_{k}^{F_{0}}$ are computed by analytically differentiating Eq. 9.

In the continuous adjoint of PUMA, the aeroelastic adjoint development is largely similar. Eq. 4 is further extended by a discrete part containing the structural adjoint variables and variation in the residuals of the discrete CSM equations ("hybrid" continuous-discrete FSI adjoint), i.e.
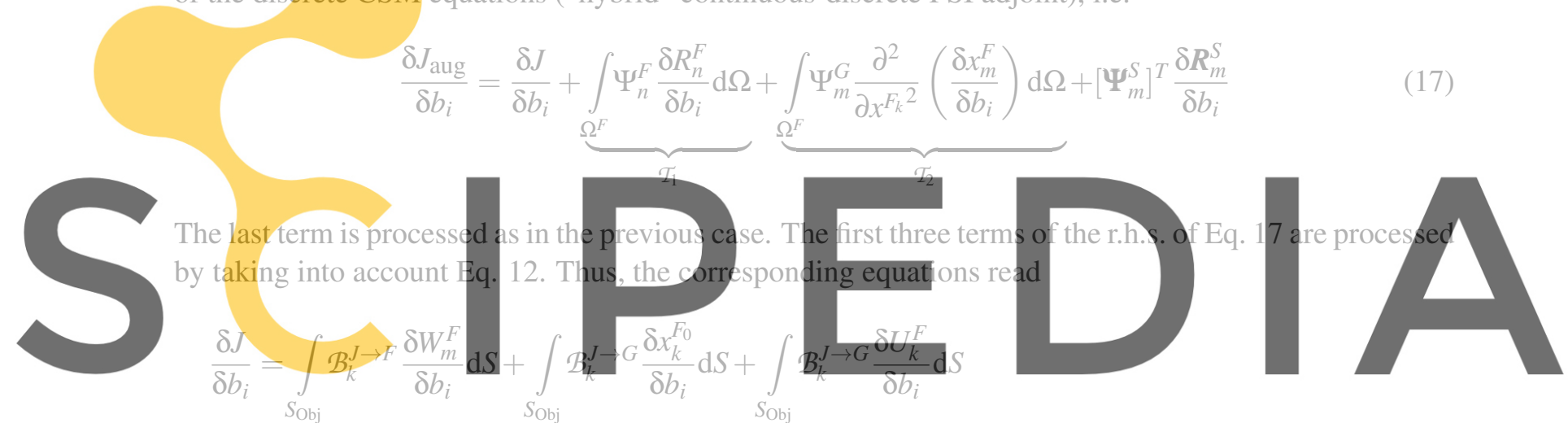

Register for free at httpsw/Awww.sciped

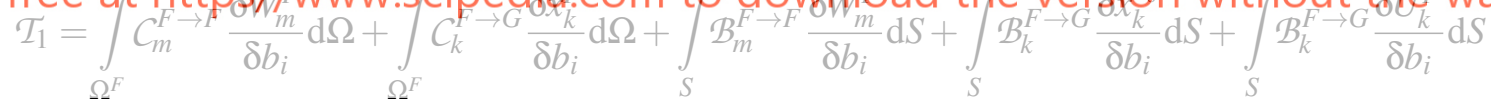

$$
\mathcal{T}_{2}=\int_{\Omega^{F}} C^{G \rightarrow G} \frac{\delta x_{k}^{F}}{\delta b_{i}} \mathrm{~d} \Omega+\int_{S} \mathcal{B}_{k}^{G \rightarrow G} \frac{\delta x_{k}^{F_{0}}}{\delta b_{i}} \mathrm{~d} S+\int_{S} \mathcal{B}_{k}^{G \rightarrow G} \frac{\delta U_{k}^{F}}{\delta b_{i}} \mathrm{~d} S
$$

Integrals containing variations in $U^{F}$ are discretized and then combined with those containing variations of $\boldsymbol{U}^{F}$ arising from the last term of Eq. 17, effectively leading to the discrete expression of the adjoint structural displacements on the CFD surface mesh. The adjoint boundary conditions on the CFD domain are also different, since now the third integral in the second of equations Eq. 18 has to be discretized and, then, combined with terms arising from the variations of $\boldsymbol{f}^{S}$ hidden in the variations of $\boldsymbol{R}^{S}$. Thus, the adjoint field equations are exactly the same as in the single-discipline case and the only difference (from the adjoint to the CFD point of view) is the new discrete expression of adjoint boundary conditions. Compared to the single-discipline case, the final expression of SDs is augmented only by a term same as the last term of Eq. 16. 


\section{VERIFICATION OF SENSITIVITY DERIVATIVES}

The SDs computed by the adjoint of AETHER and PUMA, with and without aeroelastic effects, are compared to central FDs. Moreover, in order to quantify the accuracy loss due to the commonly used "frozen" turbulence assumption, the adjoint sensitivities are also computed by neglecting variations in turbulent quantities w.r.t. the design parameters. It is shown that, when including the turbulence model in the adjoint, the accuracy of SDs is significantly improved for both CFD tools and, hence, this approach is followed in all optimization runs, see Fig. 5.
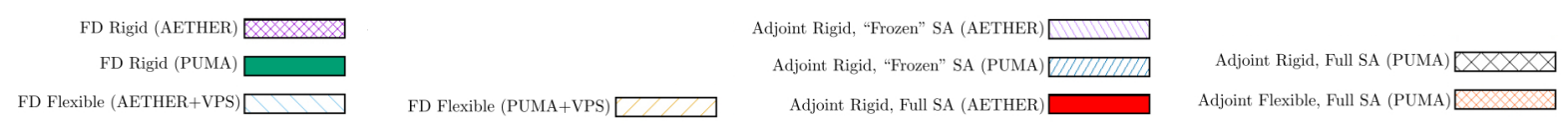

Adjoint Rigid, Full SA (AETHER)

Adjoint Flexible, Full SA (PUMA)
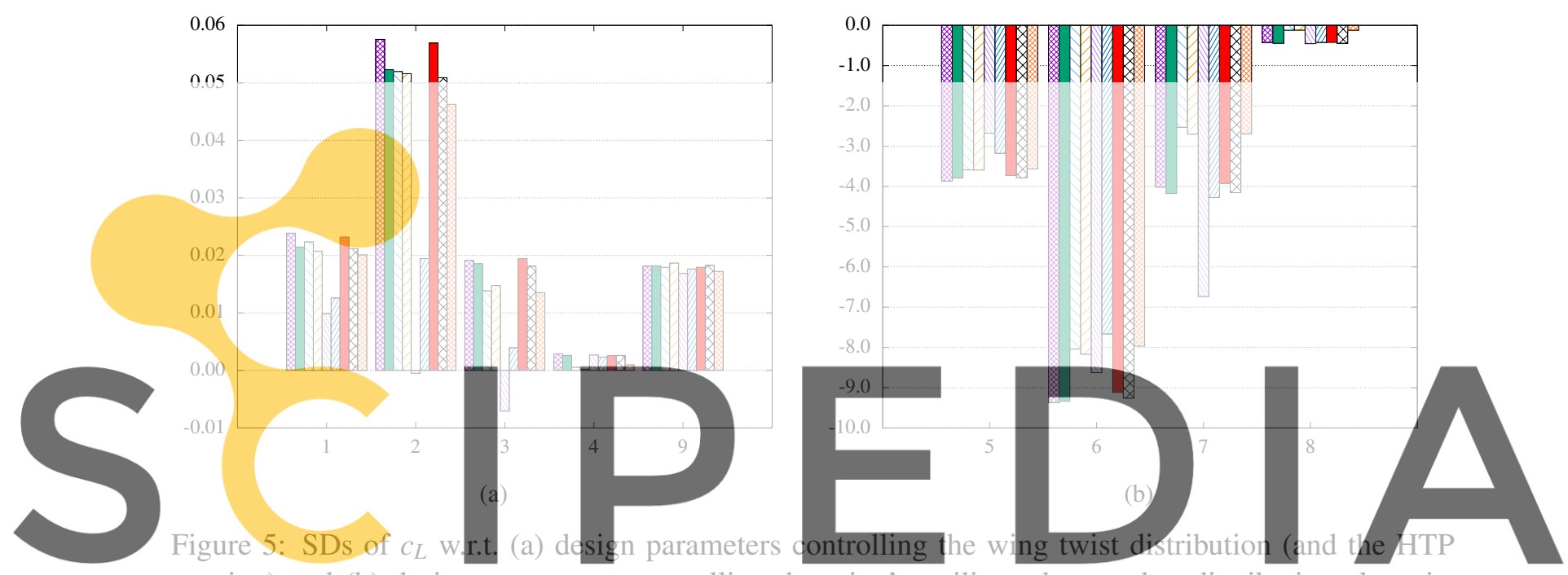

rotation) and (b) design parameters controlling the wing's trailing edge camber distribution along its

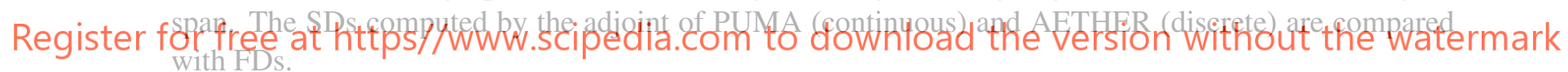

\section{OPTIMIZATION RESULTS}

Two optimization runs have been performed for optimizing the GBJ geometry, by considering or neglecting wing structure flexibility. The shape parameterization model is the one described in Section 4. Flow conditions correspond to $M_{\mathrm{inf}}=0.82$, angle of attack of $2.5^{\circ}$ and a flight altitude of $41000 \mathrm{ft}$. In both cases, the SLSQP algorithm [20] is used to drive the search of the design space and PUMA is used as the primal and adjoint CFD tool. Both optimization runs target in minimizing the drag coefficient $\left(c_{D}\right)$, with the lift coefficient $\left(c_{L}\right)$ greater than or equal to that of the datum geometry (with a 0.01 threshold) and the absolute value of the pitching moment coefficient $\left(\left|c_{M}\right|\right)$ less than 0.001 , to get a trimmed configuration. The convergence history of the rigid wing optimization is presented in Fig. 6a. In the second optimization run, the structural response of the wing structure was taken into account in the evaluation of both the aerodynamic performance metrics and the corresponding sensitivity derivatives, by means of the coupled adjoint workflow of Section 5. The hybrid continuous(flow)-discrete(structure) approach employed in 
the PUMA + VPS workflow is used. The optimization convergence history is presented and results are commented in Fig. 6b.
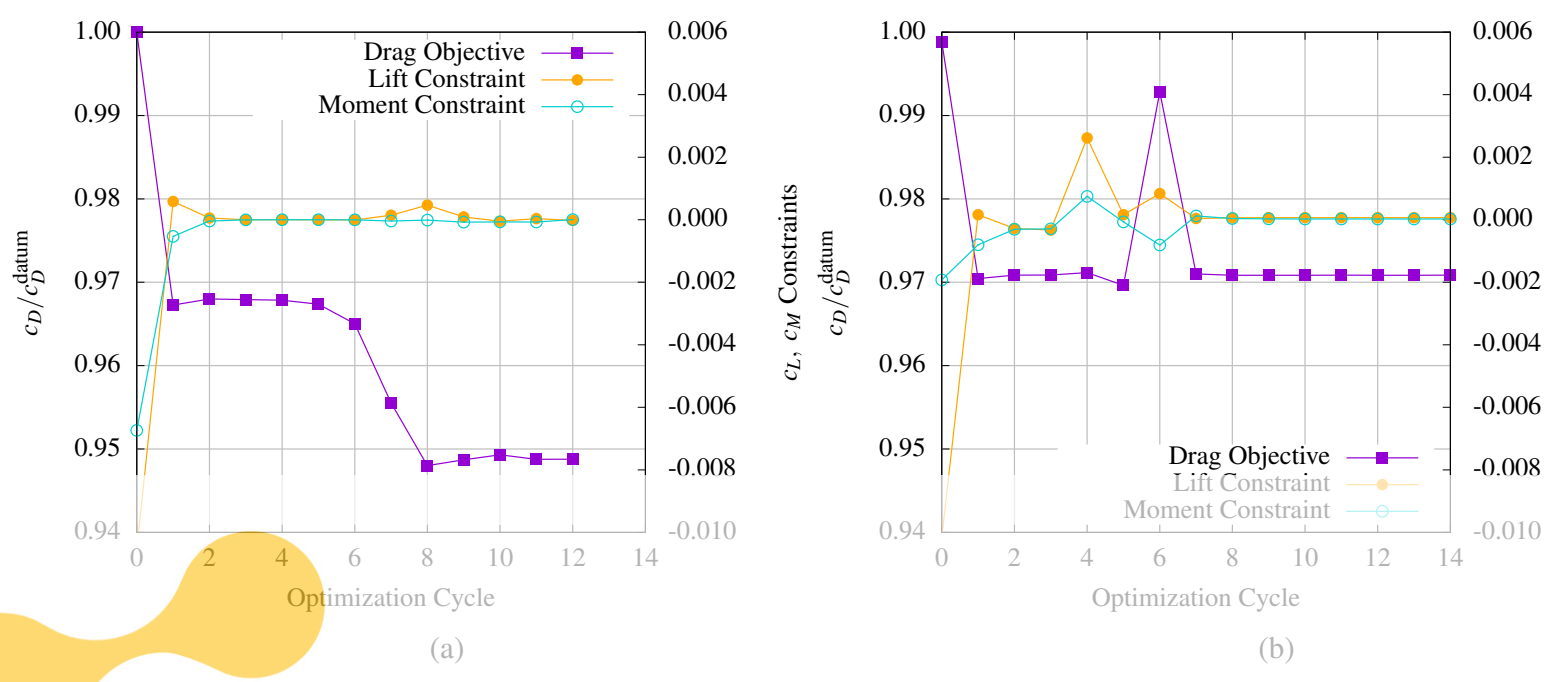

0.006

.004

Figure 6: (a) Convergence of the rigid wing optimization. A solution satisfying both aerodynamic constraints is obtained after 12 cycles with $\sim 5 \%$ reduction in $c_{D}$, compared to the datum shape. Reevaluating this design with an aeroelastic tool, the $c_{D}$ reduction was $4.7 \%$ and the pitching moment

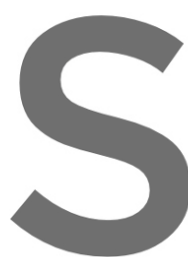
constraint was violated. constraints was obtained tum shape. Since, the aeroelastic criteria.
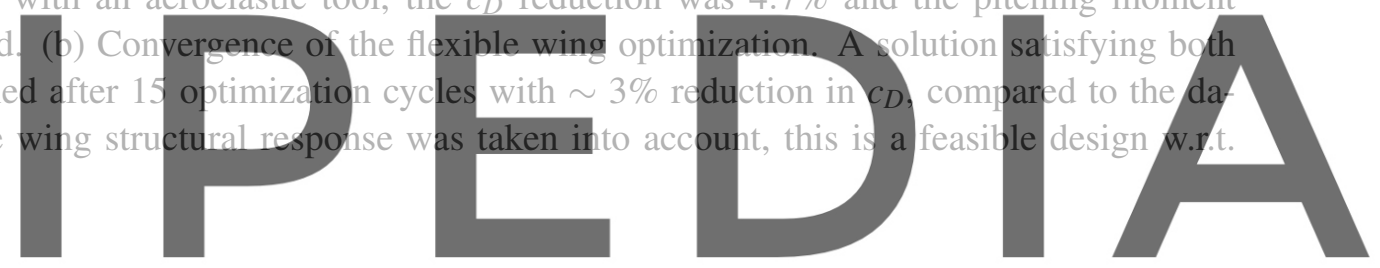

The design parameters corresponding to the rigid optimum are compared to those of the flexible

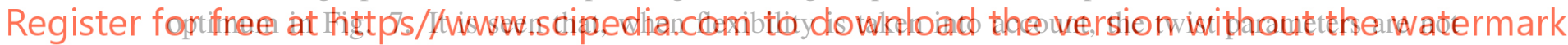
varied significantly, since large variations lead to constraint violation. In Fig. 8, the pressure coefficient fields on the suction side of the datum, single-discipline optimum and multi-disciplinary optimum are compared. For the former, drag reduction is mostly achieved by reducing the shock intensity. This not so evident in the latter. Moreover, it can be seen that the loading of the wing at the winglet area is significantly increased for the flexible optimum.

\section{CONCLUSIONS}

The development of the adjoint method for computing aeroelastic sensitivities of a business jet flexible wing was presented. Two CFD codes (AETHER and its discrete adjoint and PUMA and its continuous adjoint) were coupled with a CSM FE model for the wing structure (VPS). Optimization runs show that neglecting aeroelastic effects during the optimization leads to potentially infeasible designs. Thus, the importance of the MDO approach for this class of optimization problems is convincingly demonstrated. The cost of computing coupled SDs is roughly $3 x$ the cost of performing a single-discipline aerodynamic analysis and sensitivity derivative computation. Differences caused by the "frozen" turbulence assumption in an adjoint code are also demonstrated. On-going work focuses on the extension of the MDO 


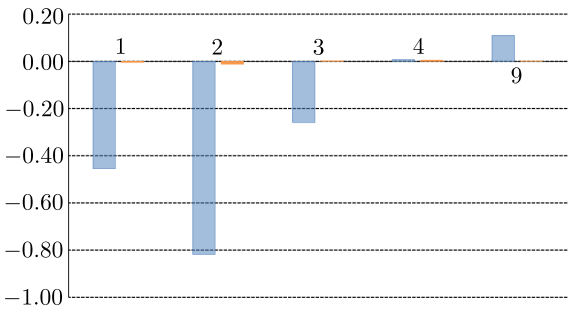

(a)

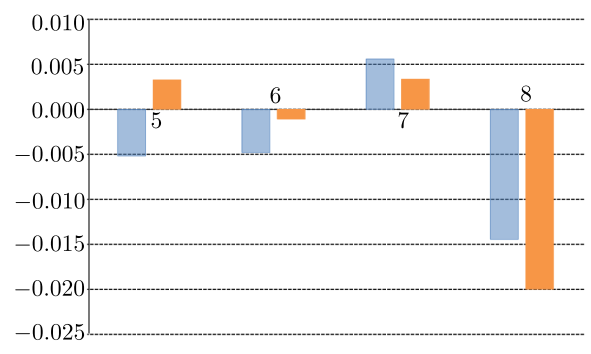

(b)

Rigid Optimum

Flexible Optimum

Figure 7: Comparison of the optimal set of (a) twist (parameters 1,2,3,4 and 9) and (b) trailing edge camber (parameters 5,6,7,8) design parameters located from the rigid and flexible optimizations.

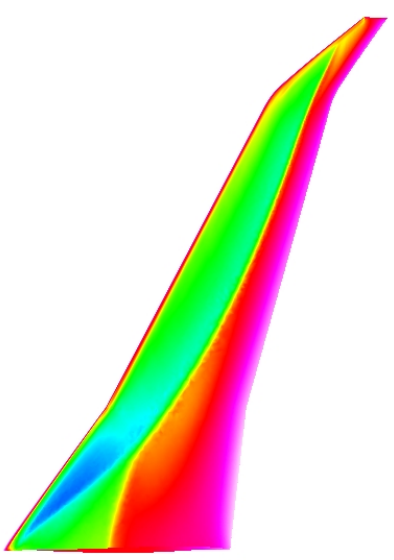

(a)

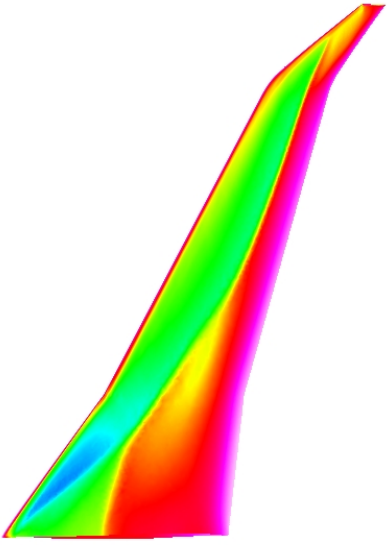

(b)

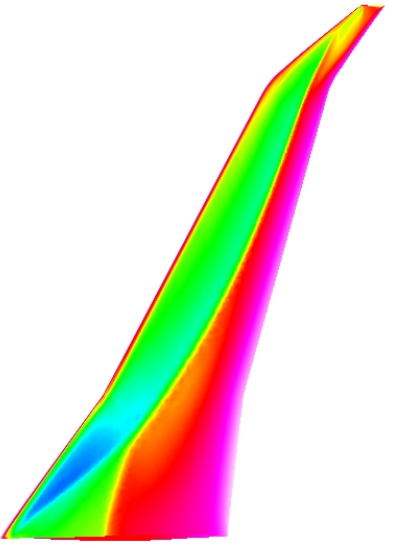

(c)

Figure 8: Comparison of the pressure coefficient iso-areas along the suction side surface of the wing of the (a) datum, (b) rigid and (c) flexible optimal airframe.

framework to include variations in the structural model properties into the optimization process.

\section{ACKNOWLEDGMENT}

This project has received funding from the European Union's Horizon 2020 research and innovation programme under grant agreement No 769025 (MADELEINE). The authors would like to thank Dr. Olivier Amoignon for his contribution in the development of the wing structure FE model and the RBFbased non-matching interface tool.

\section{REFERENCES}

[1] Adelman, H., Haftka, R. Sensitivity analysis of discrete structural systems. AIAA Journal, (1986), 24(5):823-832.

[2] Jameson, A., Reuther, J. Control theory based airfoil design using the Euler equations. $5^{\text {th }}$ Symposium on Multidisciplinary Analysis and Optimization, (1994), Panama City Beach, Florida, USA. 
[3] Giles, M., Pierce, N. Adjoint equations in CFD - Duality, boundary conditions and solution behaviour. 13 ${ }^{\text {th }}$ CFD Conference, (1997), Snowmass Village, CO, USA.

[4] Gray, J., Hwang, J., Martins, J., Moore, K., Naylor, B. OpenMDAO: An open-source framework for multidisciplinary design, analysis, and optimization. Struct. Multidiscipl. Optim., (2019), 59(4):1075-1104.

[5] Burghardt, O., Gauger, N., Gomes, P., Palacios, R., Kattmann, T., Economon, T. Coupled Discrete Adjoints for Multiphysics in SU2. AIAA AVIATION 2020 FORUM, (2020).

[6] Hughes, T., Franca, L., Mallet, M. A New Finite Element Formulation for Computational Fluid Dynamics: I Symmetric Forms of the Compressible Euler and Navier Stokes Equations and the Second Law of Thermodynamics. Comput. Methods Appl. Mech. Eng., (1986), 54:223-234.

[7] Chalot, F. Industrial aerodynamics. In Stein, E., de Borst, R., Hughes, T., editors, Encyclopedia of Computational Mechanics, (2004), John Wiley \& Sons.

[8] Kampolis, I.C., Trompoukis, X.S, Asouti, V.G. and Giannakoglou, K.C. CFD-based analysis and two-level aerodynamic optimization on Graphics Processing Units. Comput. Methods Appl. Mech. Eng. (2010) 199:712-722.

[9] Spalart, P. and Allmaras, S. A one- equation turbulence model for aerodynamic flows. La Recherche Aérospatiale (1994) 1:5-21.

[10] Martin, L., Rogé, G. Calcul de la sensibilité d'ordre 2 d'une observation aérodynamique, ESAIM Proc, (2009), 27:138-155.

[11] Hascoët, L. TAPENADE: a Tool for Automatic Differentiation of programs. $4^{\text {th }}$ ECCOMAS, (2004), Jyvaskyla, Finland.

[12] Zymaris, A., Papadimitriou, D., Giannakoglou, K., Othmer, C. Continuous adjoint approach to the Spalart-Allmaras turbulence model for incompressible flows. Comput. Fluids (2009), 38(8):15281538.

[13] Bueno-Orovio, A., Castro, C., Palacios, F., Zuazua, E. Continuous adjoint approach for the SpalartAllmaras model in aerodynamic optimization. AIAA Journal (2012), 50(3):631-646.

[14] Shroff, G. and Herbert, B. Stabilization of unstable procedures: The Recursive Projection Method. SIAM Journal on Numerical Analysis (1993), 40(4):1099-1120.

[15] Rogé, G., Kleiveld, S., Loyatho, X., Daumas, L. Shape optimization for greener aircraft: the Dassault Aviation vision and perspectives. ECCOMAS Congress (2016), Crete, Greece.

[16] Kleinveld, S., Rogé, G., Daumas, L. and Dinh, Q. Differentiated parametric CAD used within the context of automatic aerodynamic design optimization. $12^{\text {th }}$ AIAA/ISSMO Multidisciplinary Analysis and Optimization Conference, (2008), Victoria, Canada.

[17] Jakobsson, S. and Amoignon, O. Mesh deformation using Radial Basis Functions for gradientbased aerodynamic shape optimization. Comput. Fluids (2007) 36:1119-1136.

[18] Farhat, C., Lesoinne, M. and LeTallec, P. Load and motion transfer algorithms for fluid/structure interaction problems with non-matching discrete interfaces: Momentum and energy conservation, optimal discretization and application to aeroelasticity. Comput. Methods Appl. Mech. Eng. (1998) 157:95-114.

[19] Degroote, J., Haelterman, R., Annerel, S., Bruggeman, P., Vierendeels, J. Performance of partitioned procedures in fluid-structure interaction. Comput. Struct. (2010), 88:446-457.

[20] Kraft, D. A software package for sequential quadratic programming. Tech.Rep. DFVLR-FB 88-28, (1988), DLR German Aerospace Center - Institute for Flight Mechanics, Koln, Germany. 